\title{
Teleoperación de un vehículo remoto en un medio de acceso inalámbrico mediante el uso de una interfaz háptica
}

Remote vehicle teleoperation through a haptic interface

Arys Carrasquilla Batista'

Fecha de recepción: 20 de enero del 2012 Fecha de aprobación: 19 de marzo del 2012

Carrasquilla, A. Teleoperación de un vehículo remoto en un medio de acceso inalámbrico mediante el uso de una interfaz háptica. Tecnología en Marcha.Vol. 25, Nº 5. Pág 5-I3. 


\section{Resumen}

La teleoperación permite que el ser humano pueda llevar a cabo ciertas tareas en lugares muy lejanos, de difícil acceso o de condiciones hostiles para la presencia de un operador. Con este proyecto se logró teleoperar un vehículo remoto por medio del protocolo de comunicación Bluetooth, para lo cual se adaptó una interfaz háptica (Novint Falcon). Desde la interfaz, el operador puede enviar la consigna de movimiento $y$, además, obtener sensaciones conforme a la información de los sensores incluidos al sistema. Como vehículo remoto se utilizó un Lego Mindstorms con capacidad de comunicación Bluetooth, al cual se incorporó un sensor de contacto y otro de ultrasonido con el fin de percibir en la interfaz háptica la retroalimentación de fuerzas. A una computadora estándar se le dio capacidad de comunicación Bluetooth por medio de un adaptador USB, desde la cual se ejecuta un programa creado en $\mathrm{C}++$ para controlar las acciones de la interfaz háptica, enviar los comandos de movimiento al vehículo y recibir la información de los sensores, la cual reproduce sensaciones al operador.

\section{Palabras clave}

Teleoperación, protocolo Bluetooth, interfaz háptica, USB dongle, $\mathrm{C}++$.

\begin{abstract}
Teleoperation allows human beings to carry out certain tasks in places far away, inaccessible or with hostile conditions for the presence of an operator.

In this project a remote vehicle is teleoperated using a bluetooth wireless connection, to accomplish this an haptic interface (Novint Falcon) was used. The operator can give movement instructions to the vehicle and obtain sensations, according to the information received from the sensors connected to the system.

The remote vehicle was a Lego Mindstorms with bluetooth communication capabilities, a touch sensor and ultrasonic sensor were included in order to perceive reflection of forces through the haptic interface.
\end{abstract}

A USB adapter for bluetooth communication was added to a standard computer; a program in $\mathrm{C}++$ is executed over this computer to control the haptic interface, send movement commands to the vehicle and receive sensor information to reproduce sensations to the operator.

\section{Key words}

Teleoperation, Bluetooth protocol, Haptic interface, USB dongle, $\mathrm{C}++$. 


\section{Introducción}

Desde la antigüedad, el hombre ha desarrollado diferentes técnicas y herramientas para incrementar su capacidad al manipular objetos; por ejemplo, es de vital importancia para un herrero utilizar pinzas cuando realiza sus trabajos con materiales candentes, peligrosos para el ser humano (Gómez, Ollero y García, 2006).

Existen actividades que pueden provocar riesgos en la salud de las personas, como es el caso de los procesos radiactivos, o en medios inhóspitos de trabajo, donde no es posible contar con un operador de forma local; por esta razón surge, a mediados del siglo $X X$, una nueva tecnología llamada "teleoperación", la cual consta de un sistema de telecomunicaciones que permite distancia entre el trabajador y sus labores. Este campo de acción ha crecido notablemente, con aplicaciones que van desde el entretenimiento hasta el rescate de personas en peligro (Gómez et ál., 2006; Sheridan, 1993).

La teleoperación se puede ver como la extensión de las capacidades sensoriales y de la destreza humana a un sistema remoto, con diferentes formas de intervención del operador según su campo de acción.

Entre las tecnologías actuales para interfaces de teleoperación, se encuentran las interfaces hápticas, diseñadas para enviar la consigna de movimiento ingresada por el usuario, así como para recibir la retroalimentación necesaria para dar sensaciones de fuerza o tacto. Cada interfaz puede variar su complejidad según sus características principales: número de grados de libertad, calidad, precisión, rangos de desplazamientos y fuerzas (Pinto, 2009).

En el Laboratorio de Investigaciones en Robótica y Automatización (LIRA) de la Escuela de Ingeniería Electrónica del Instituto Tecnológico de Costa Rica, se cuenta con interfaces hápticas y sistemas robotizados que han permitido incursionar en proyectos sobre esta rama de la tecnología, como, por ejemplo, la teleoperación a través de Bluetooth, Wi-Fi e internet. En este proyecto en particular se explicará el desarrollo de la teleoperación mediante Bluetooth.

\section{Materiales y métodos}

\section{Teleoperación}

La teleoperación consta de cinco partes fundamentales:

I. Operador: es la persona encargada de tomar el control de la operación a distancia.

2. Interfaz: permite al operador ingresar la forma en que desea que se comporte el dispositivo remoto.

3. Controles y canales de comunicación: se encargan de comunicar la zona remota y local, por ello modulan, transmiten y adaptan el conjunto de señales.

4. Dispositivo teleoperado: existen distintas clases de estos dispositivos, como actuadores, vehículos, robots, entre otros.

5. Sensores: recolectan información de forma local y remota para generar la retroalimentación necesaria (Sheridan, 1993; Butner y Ghodoussi, 2003).

\section{Interfaces hápticas}

Se denomina interfaz háptica al conjunto de dispositivos que permiten a su operador sentir y manipular objetos en entornos virtuales o en sistemas teleoperados. Con este tipo de tecnología, se le da al usuario la capacidad de interactuar con el entorno; la transmisión de información es bidireccional (en ambos sentidos) y en tiempo real (Gómez, Ollero y García, 2006; Monasterio-Huelin, s.f.; Arias, 2008). En la figura I se observa la interfaz háptica Novint Falcon utilizada en este proyecto.

A continuación se mencionan sus principales características técnicas:

- Espacio de trabajo tridimensional de $4 \times 4 \times 4$ pulgadas.

- Capacidad de fuerzas de dos libras.

- Resolución de posición de 400 dpi.

- Tiempo de un segundo para desconexión rápida del dispositivo.

- Comunicación USB 2.0.

- Tamaño físico de 9×9×9 pulgadas.

- Peso de seis libras. 
- Potencia de 30 W, 100-240 V, 50-60 Hz.

- Programable por medio del SDK (Software Development Kit), provisto por el fabricante (Novint, s.f.).

\section{Bluetooth}

Es una tecnología que facilita las comunicaciones móviles, ya que interconecta dispositivos y posibilita la conexión a internet inalámbrica; es de pequeña escala, bajo costo y utiliza enlaces de radio de corto alcance. Opera en la banda de $2,4 \mathrm{GHz}$ con capacidad de atravesar paredes, lo que la hace ideal tanto para operaciones móviles como de oficina.

La frecuencia de trabajo se encuentra en el rango de los 2,4 a 2,48 GHz, con hasta 79 saltos de frecuencia de un | Mhz, que puede variar según la región, ya

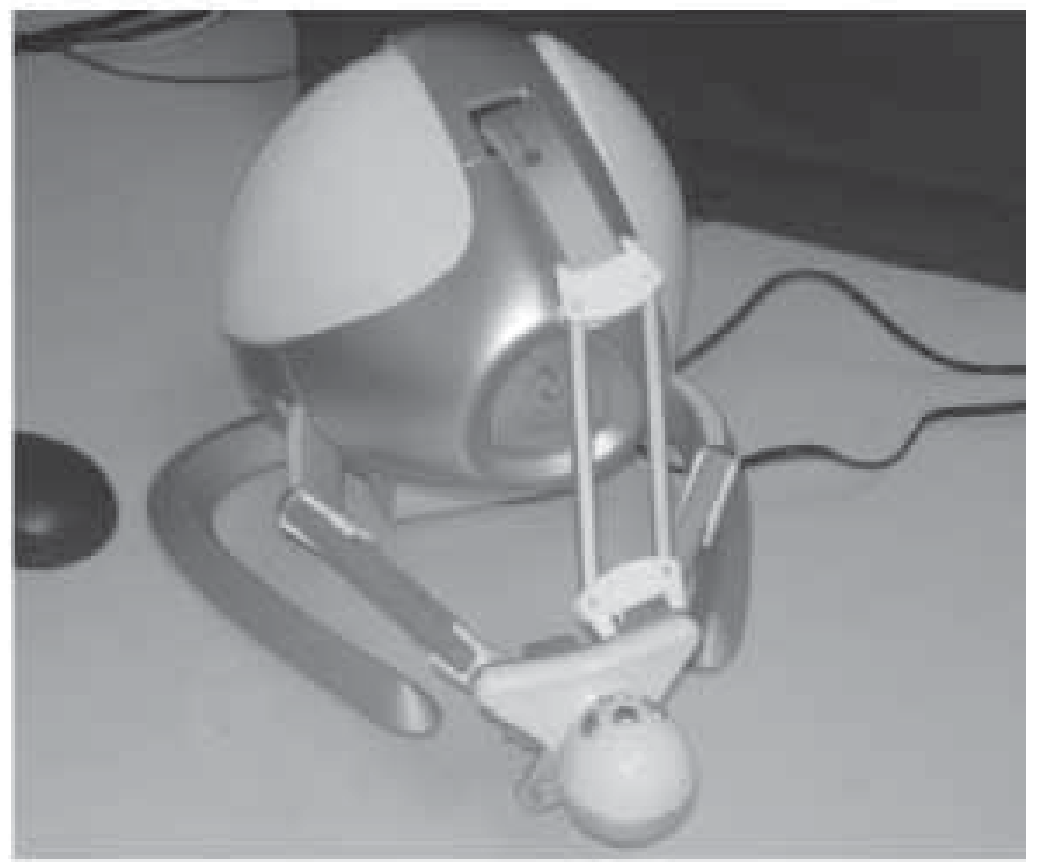

Figura I. Interfaz háptica Novint Falcon.
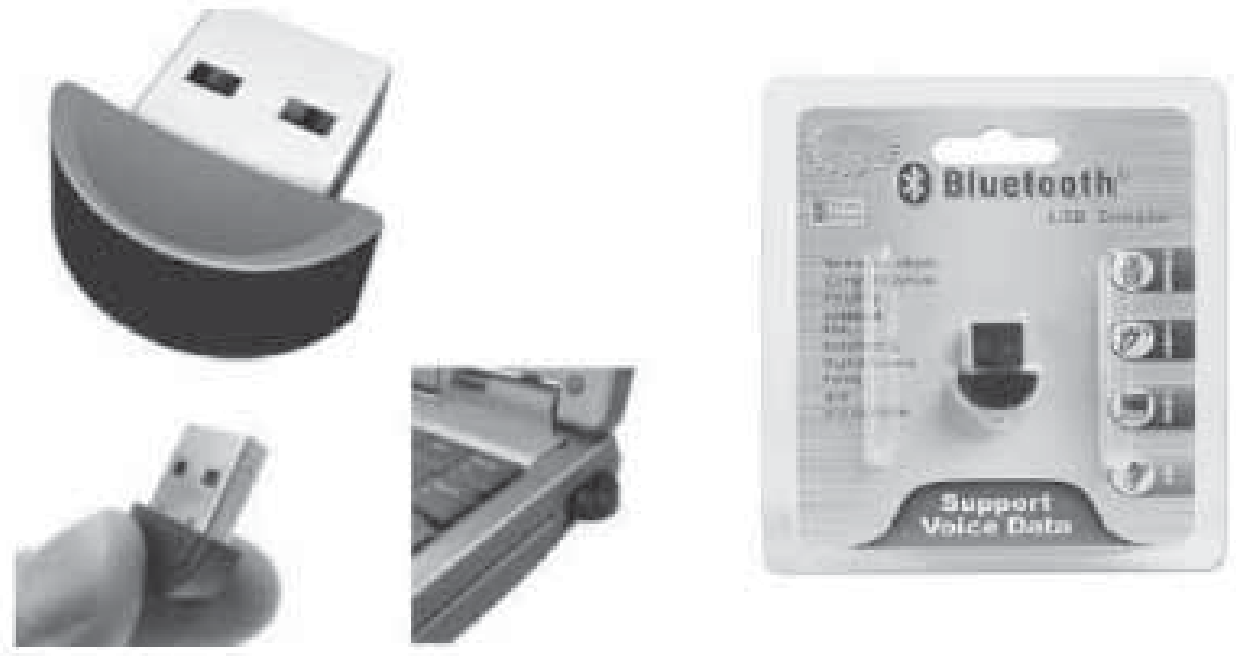

Figura 2. Bluetooth USB dongle. 
que posee capacidad de transmitir en full duplex con un máximo de 1600 saltos/seg. De acuerdo con el tipo de versión y clase del dispositivo, así varían su capacidad de canal, alcance y potencia de salida.

El USB dongle permite dotar de comunicación Bluetooth a todas aquellas computadoras con algún puerto USB disponible que no incorporen esta tecnología. En la figura 2 se muestra el USB dongle utilizado en este proyecto; se puede notar la pequeña escala del dispositivo (Bluetooth SIG Inc., 20I I; Bluetooth SIG, s.f.; Figuera, s.f.; Pérez, s.f.; Rodríguez y Maya, 2004.

\section{Lego Mindstorms}

El Lego Mindstorms es un producto de la empresa Lego, que permite armar y programar una gama muy amplia de estructuras predefinidas o de invención propia del consumidor.

El kit del Lego Mindstorms proporciona un software de programación que utiliza un ambiente de bloques gráficos. En este proyecto fue necesario recurrir a documentos especializados, brindados por el fabricante, con el fin de tener acceso a un conjunto de comandos e instrucciones más complejos que permitieran incorporar el estándar de comunicación Bluetooth (The Lego Group, 20I2).

\section{Descripción del sistema inalámbrico teleoperado con retroalimentación de posición mediante una interfaz háptica}

En la figura 3 se observa el diagrama general de la solución implementada.

Este sistema es capaz de tener una comunicación bidireccional entre la interfaz háptica y el vehículo remoto. Para lograr esto, primeramente, se tiene la interfaz háptica comunicada con la PC por medio del protocolo de comunicación USB; luego, dicha PC se comunica por medio de un puerto USB al dispositivo Bluetooth USB dongle, el cual permite la comunicación Bluetooth con el vehículo remoto. La consigna de movimiento introducida en la interfaz háptica por el usuario llega al vehículo remoto para ser accionado, y este, dependiendo de su interacción con el entorno, brinda la información necesaria para ser procesada por la PC, de forma que se puedan generar sensaciones en la interfaz háptica.

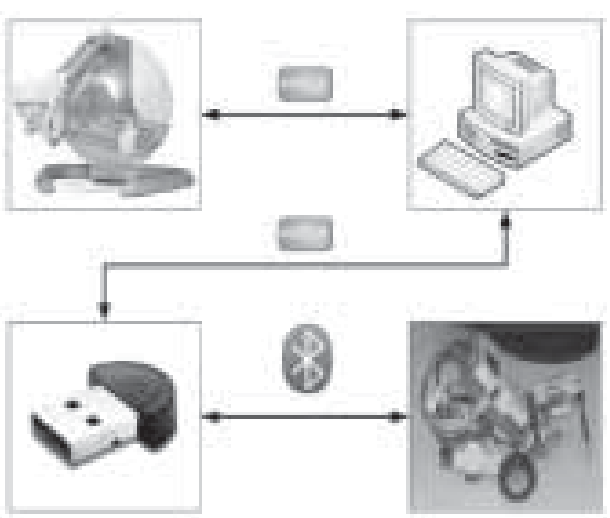

Figura 3. Diagrama general del sistema teleoperado.

\section{Vehículo remoto}

En la figura 4 se muestra el vehículo remoto implementado de Lego Mindstorms.

El vehículo remoto permite, mediante dos servomotores con regulación de potencia, movimiento hacia adelante, atrás, izquierda y derecha. Cabe destacar que para poder dar movimiento hacia sus laterales (izquierda o derecha) se acciona el servo que se encuentra en el lado que se quiere girar mientras el otro se encuentra detenido, así la fricción causada hace girar en uno u otro sentido el vehículo.

Se puede notar cómo el vehículo remoto incorpora dos sensores: contacto y ultrasonido. Mediante el sensor de contacto se puede detectar el momento que el vehículo se encuentra avanzando hacia adelante y choca con un obstáculo; mientras que el ultrasónico permite detectar cuando se tiene un obstáculo en su parte trasera y la distancia al que se encuentra, lo que evita una colisión.

\section{Bluetooth USB dongle}

Se debe destacar que, para el uso de este dispositivo, al conectar en un puerto USB automáticamente se instalarán los drivers necesarios para su uso, de la misma forma que cuando insertamos un dispositivo de almacenamiento masivo en un puerto de este tipo. El adaptador USB dongle tiene la particularidad de dar comunicación Bluetooth y, además, hace funcionar el puerto USB al que se conecta como un puerto COM, mediante el SPP (Serial Port Profile). 


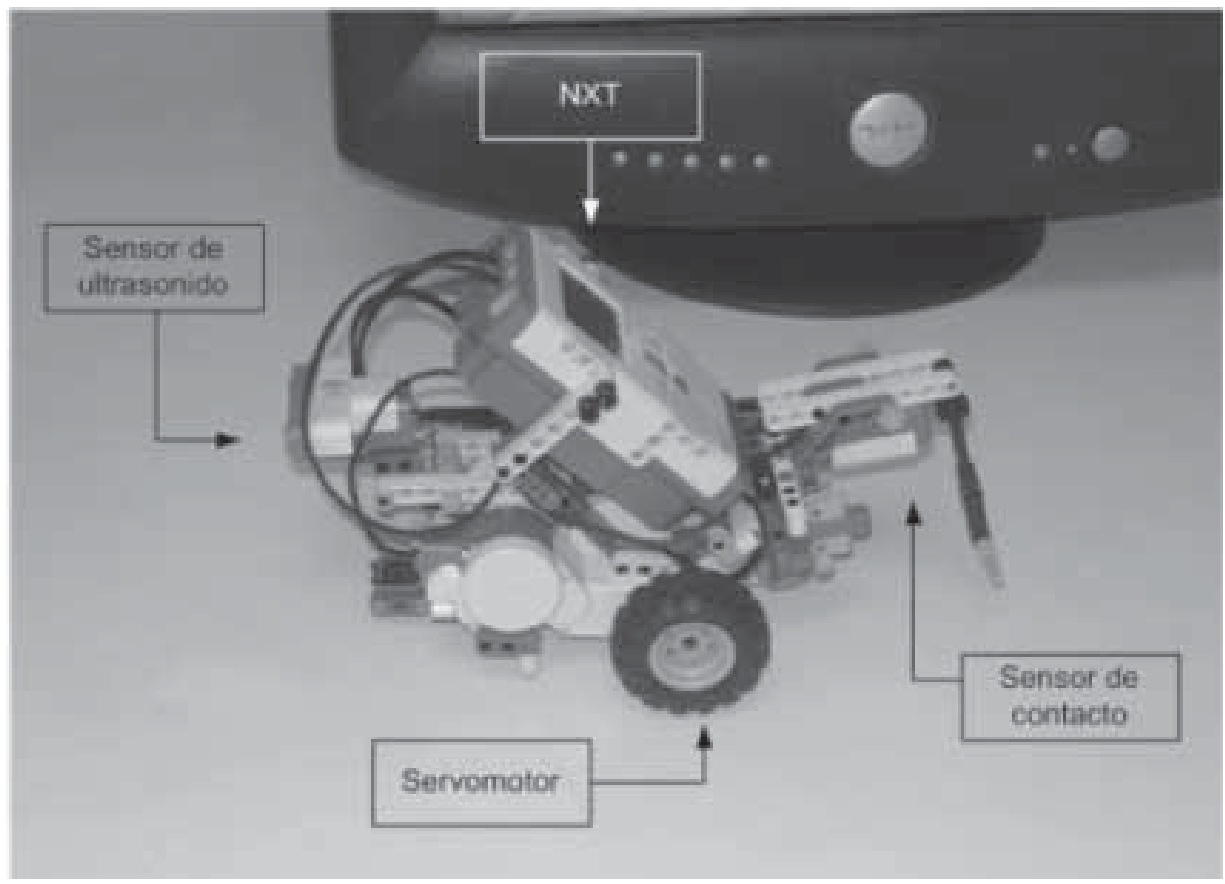

Figura 4. Vehículo remoto construido.

\section{Rutinas implementadas desde la PC para el vehículo remoto}

Aquí se describe la rutina general implementada en la PC para manejar todo el sistema teleoperado; en la figura 5 se muestra la forma en que se tomó la consigna de movimiento para el vehículo remoto desde la interfaz háptica, en un cubo de lado de 500 ul (unidades lineales, debido que se trata de un redimensionamiento a nivel de software).

La manera en que se toma la consigna de movimiento depende de la posición del mando de la interfaz háptica. El primer detalle importante a mencionar es la forma en que se le indica la magnitud de la potencia de movimiento al vehículo remoto, para lo que se utiliza el eje Z: entre más se encuentre el mando en $-Z$, mayor será la potencia asignada, hasta alcanzar un $100 \%$ del valor permitido por el NXT; por el contrario, entre más se encuentre el mando en $+Z$, menor será la potencia asignada, hasta llegar a cero, exactamente cómo se comporta el pedal de un automóvil.

Para dar una orden al NXT de movimiento de giro hacia la derecha, la posición del mando de la interfaz debe estar sobre las 200 ul del eje $X_{\text {; de }}$ modo similar, es posible dar un giro a la izquierda cuando la interfaz se posiciona en menos de $-200 \mathrm{ul}$ en el eje
X. Es el mismo procedimiento para el avance hacia delante y hacia atrás, tomando en cuenta la posición en el eje Y. La zona central del cubo es neutra y no se asignan movimientos.

En la figura 6 se muestra el diagrama de flujo de la rutina general implementada para el sistema teleoperado.

La rutina general implementada en la PC para manejar todo el sistema teleoperado cuenta con rutinas especiales para cada función: interfaz háptica, conexión Bluetooth, manejo de servomotores y sensores.

La rutina comienza con cuatro llamadas a otras subrutinas:

I. Inicializar la interfaz háptica: al realizar esta acción se habilita la función "hilo", ejecutada cíclicamente, la cual permite tener el valor de las coordenadas de la interfaz háptica, reproducir fuerzas y activar banderas (freno de emergencia y terminar programa).

2. Establecer el enlace de comunicación Bluetooth con el vehículo remoto.

3. Configurar el sensor de contacto.

4. Configurar el sensor de ultrasonido. 


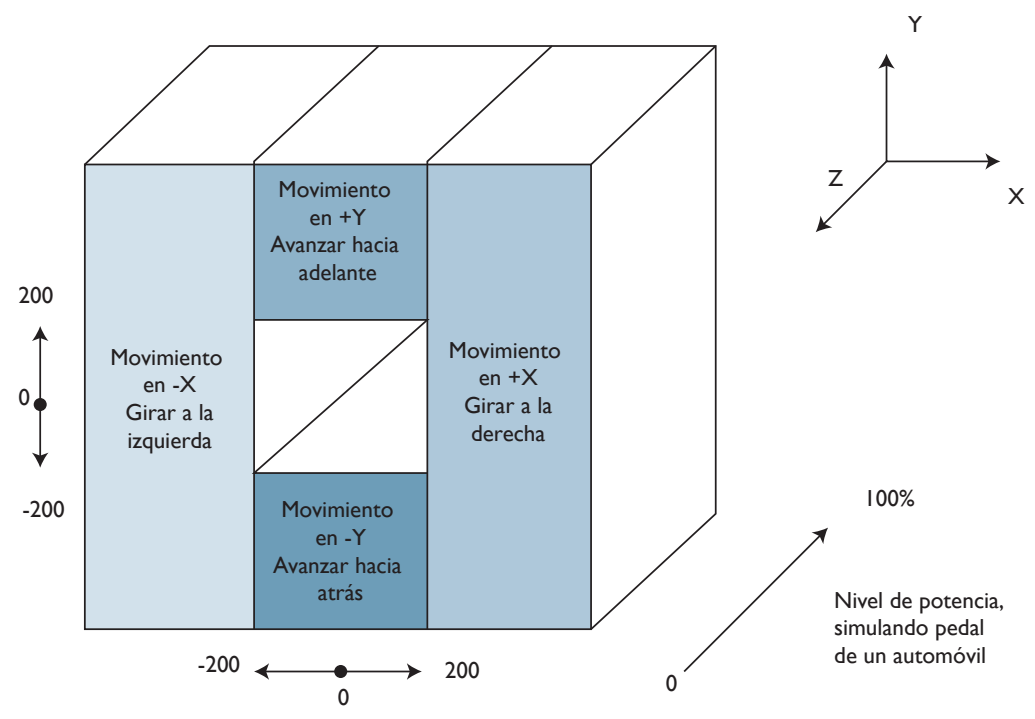

Figura 5. Diagrama de la forma en que se toma la consigna de movimiento desde la interfaz háptica para el vehículo remoto.

Se procede a entrar en un ciclo hasta que se levante la bandera de terminar programa; si se cumple esta condición se hace uso de la rutina para apagar los servomotores y salir de la aplicación; en caso contrario, se procede a cinco pasos más dentro de la subrutina, mencionados con más detalle a continuación:

1. Con respecto a la posición del eje $Z$ de la interfaz háptica, se procede a calcular la potencia que será parte de la consigna de movimiento en el momento que haya que accionar algún servomotor.

2. Se lee el valor del sensor de contacto.

3. Según el valor leído en el paso anterior, se levanta la bandera de obstáculo delantero, necesaria en la función de cálculo de sensaciones hápticas.

4. Se lee el sensor de ultrasonido.

5. De acuerdo con el valor leído en el paso anterior, se determina si en la parte trasera del automóvil se tiene un objeto a menos de $10 \mathrm{~cm}$, para así levantar la bandera de obstáculo trasero, necesaria en la función de cálculo de sensaciones hápticas.

Luego se envía la consigna de movimiento, para lo que se procede a verificar las coordenadas de la interfaz háptica, como se explicó previamente en la figura 5, con lo que se determina cuál es la acción de movimiento deseada: se utiliza, entonces, la rutina de control de los servomotores, con un valor de magnitud de potencia como parámetro previamente calculado según la posición del eje $Z$.

Dependiendo el movimiento deseado, se deben encender los servomotores A y B de la siguiente forma:

- Adelante: servomotores A y B con valor de potencia calculado según eje $Z$.

- Atrás: servomotores A y B con valor de potencia calculado según eje $Z$ con signo negativo.

- Derecha: servomotor A con potencia cero y servomotor B con valor de potencia calculado según eje Z; se debe recordar que al tener un servomotor detenido y accionar el otro, la fricción provoca un giro hacia el lado del servomotor activado.

- Izquierda: servomotor A con valor de potencia calculado según eje $Z$ y servomotor B con potencia cero.

- Neutro y freno de emergencia (botón I): servomotor $\mathrm{A}$ y $\mathrm{B}$ con valor de potencia cero.

De esta forma se realiza la teleoperación del sistema y, al final, se espera que el botón 2 del mando sea presionado; de no ocurrir,el operario podrá, mediante la interfaz háptica, enviar la consigna de movimiento y, a su vez, sentir bloqueos de movimiento en el eje Y según la interacción del vehículo remoto con el medio. 


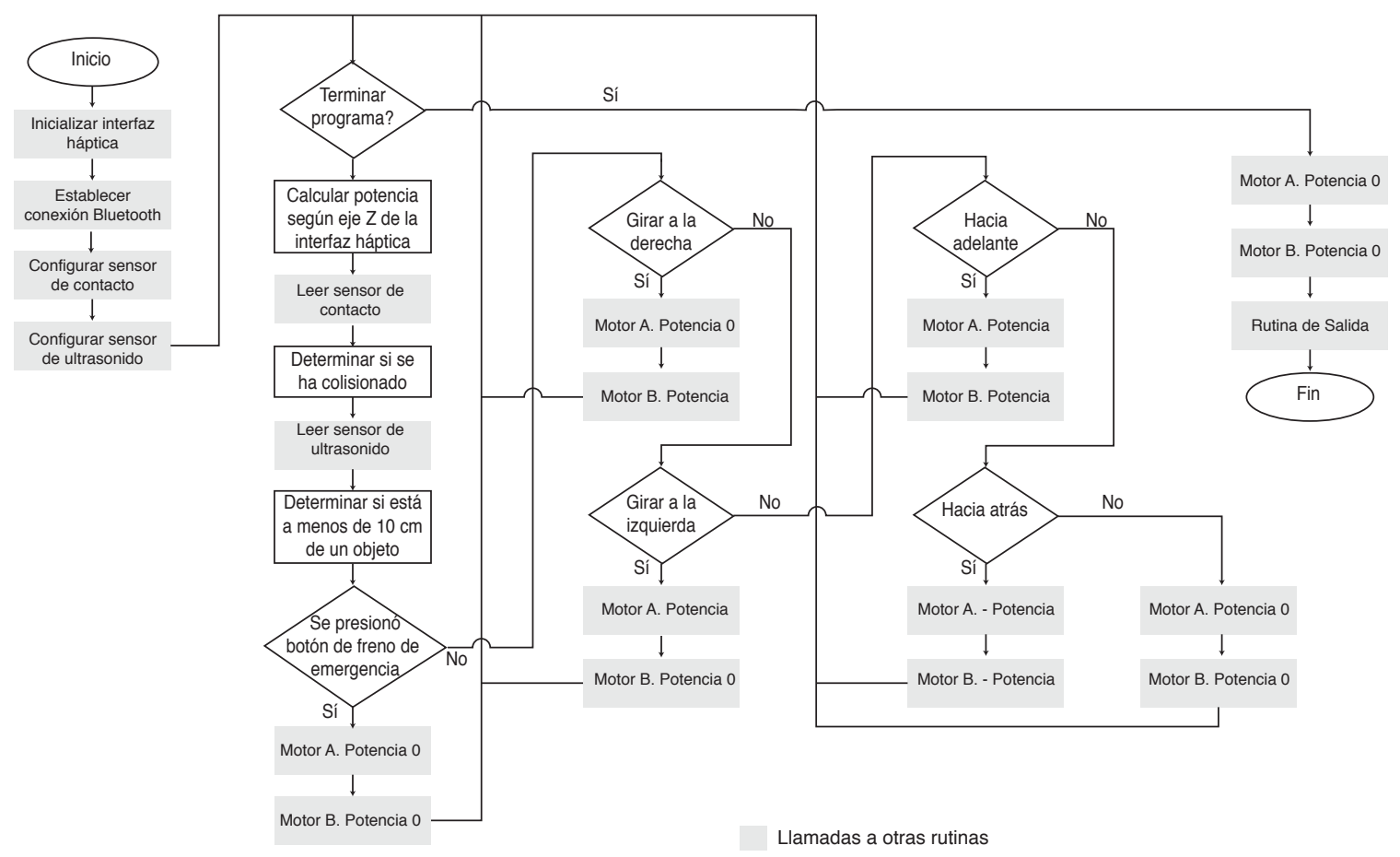

Figura 6. Diagrama de flujo de la rutina general implementada para el sistema teleoperado.

\section{Análisis de resultados}

Se logró desarrollar un sistema teleoperado de forma inalámbrica por medio de la comunicación Bluetooth, acoplando la interfaz háptica de realimentación de fuerza Novint Falcon, desde la cual el operador puede ingresar la consigna de movimiento deseada y sentir su interacción con el entorno.

A continuación se detallan los distintos experimentos realizados para comprobar la funcionalidad del sistema teleoperado, así como los resultados obtenidos en cada uno de ellos.

Pruebas de la consigna de movimiento del operador sobre el vehículo remoto: según los resultados, se verifica que la consigna de movimiento dada por el operador del sistema se refleja de manera correcta sobre el vehículo remoto. Cabe destacar que cada una de las pruebas fue realizada con diferentes valores de potencia, conforme se daba el movimiento sobre el eje Z. Se obtuvo una respuesta positiva en el $100 \%$ de los casos.

Retroalimentación del sistema teleoperado: se realizaron pruebas a distintos niveles de potencia, cuya intención era corroborar la efectividad de la retroalimentación del sistema; con este fin se registró el momento en el que el vehículo remoto detectaba un obstáculo trasero o delantero. No se obtuvo error de medición con el sensor de contacto, el cual se encontraba en la parte delantera del vehículo, ya que, cada vez que golpeaba un objeto, el sistema bloqueaba exitosamente el eje + Y, lo que impidió el avance en el eje $+Y$ al vehículo remoto.

Dos mediciones con el sensor de ultrasonido presentaron 6,67\% de error, debido a que, en ambos casos, el vehículo estaba en movimiento con valores de potencia cercanos al $100 \%$ de su capacidad y el sistema no tenía el suficiente tiempo para tomar la medición, enviar la información y reproducir la sensación háptica antes de que colisionara; de ahí que el resto de mediciones, a potencias inferiores a su límite máximo, fueran exitosas.

El sistema teleoperado mantiene un correcto funcionamiento alrededor de los $11 \mathrm{~m}$ de distancia entre la PC y el vehículo remoto, resultado predecible debido a que, si bien es cierto el USB dongle utilizado en la PC es un dispositivo Bluetooth clase I con un alcance aproximado de 100 m, el NXT no presenta la misma capacidad, ya que posee un dispositivo Bluetooth de clase 2 , con un alcance aproximado de $10 \mathrm{~m}$. 


\section{Conclusiones}

- Es posible acoplar la interfaz háptica Novint Falcon a un sistema teleoperado inalámbrico.

- El estándar de comunicación Bluetooth permite una comunicación inalámbrica con un margen de falla menor al 10\% para sistemas en movimiento.

- En sistemas teleoperados de forma inalámbrica, los tiempos de retardo se hacen notables en el sistema al aumentar la distancia y el procesamiento de información.

- El lenguaje de alto nivel $\mathrm{C}++$ permite manipular tanto el USB dongle para desarrollar aplicaciones, como el SDK de Novint.

- El sensor de ultrasonido es más sensible al nivel de potencia de movimiento que el sensor de contacto.

- Se puede construir un sistema teleoperado con retroalimentación de posición mediante una interfaz háptica y comunicación Bluetooth.

\section{Agradecimientos}

A la Escuela de Ingeniería Electrónica del Instituto Tecnológico de Costa Rica, por el espacio físico suministrado para la creación del Laboratorio de Investigaciones en Robótica y Automatización (LIRA).

A la Vicerrectoría de Investigación y Extensión (VIE), por el tiempo de investigación, suministro económico para compra de equipo y la asignación de horas asistentes.

A los estudiantes de Ingeniería Electrónica, ya hoy ingenieros, que con su tiempo y desarrollo de proyectos de graduación hicieron posible incursionar en el mundo de la teleoperación.

\section{Bibliografía}

Arias, D. (2008). Interfaces Hápticas. Tesis de Grado, Universidad Católica de Salta, Facultad de Ingeniería, Argentina. Recuperado de http://haptica.blogspot. com/2008/l0/interfaces-hpticas.html.

Bluetooth SIG, Inc. (201I) Recuperado http://www. bluetooth.com/Pages/Bluetooth-Home.aspx.

Bluetooth SIG. s.f. Especificación completa de tecnología Bluetooth según el SIG, Recuperado de https://www. bluetooth.org/spec/

Butner, S. \& Ghodoussi, M. (2003). Transforming a Surgical Robot for Human Telesurgery, IEEE Transactions on Robotics and Automation.

Figueira, J. (s.f.). Bluetooth. Protocolos y aplicaciones. Recuperado de http://neutron.ing.ucv.ve/revista-e/ No8/Jose\%20Figueira/Figueira.htm

Gómez, J., Ollero, A. \& García, A. (2006). Teleoperación y telerrobótica. Madrid: Pearson Prentice Education.

Monasterio-Huelin, F. (s.f.) Dispositivos hápticos y cirugía robótica. Recuperado de http://robolabo.etsit.upm.es/ haptico/haptico-bio- Ix2.pdf.

Novint. Recuperado de http://home.novint.com/products/ novint_falcon.php.

Pérez,J. (s.f.) Bluetooth, descripción del estándar. Recuperado de http://neutron.ing.ucv.ve/revista-e/No8/Joffre\%20 Perez/Bluetooth. I.htm.

Pinto, M. (2009). Análisis e implementación de una interfaz háptica en entornos virtuales. Tesis de grado. Facultad de Ingeniería, Universidad Nacional de Colombia, Bogotá, Colombia. Recuperado de http://www.bdigital.unal. edu.co//76////280201.2009.pdf.

Rodríguez, O. \& Maya, R. (2004). Implementación de una red inalámbrica bluetooth. Tesis de grado. Escuela de Ingeniería Eléctrica y Electrónica, Universidad del Valle, Cali, Colombia. Recuperado de http://www.univalle. edu.co/ telecomunicaciones/ trabajos_de_grado/ informes/tg_OscarRodriguez_RicardoMaya.pdf.

Sheridan, T. (1993, Oct.). Space Teleoperation through Time Delay: Review and Prognosis, IEEE Transactions on Robotics and Automation, 9 (5).

The Lego Group.Mindstorms NXTARM7 Bluetooth Interface Specification, Recuperado de http://mindstorms.lego. com/en-us/bluetooth/default.aspx

The Lego Group. Lego Mindstorms. Recuperado de http:// mindstorms.lego.com/en-us/default.aspx. 